\title{
Tipos de trabajo en las medianas empresas: estudio teórico y empírico
}

\author{
Types of work in medium enterprises: theoretical and empirical study
}

\author{
Gerardo José Zapata Rotundo \\ Ph.D., Decanato de Administración y Contaduría, Coordinación de Investigación: CIDAC, Universidad \\ Centroccidental "Lisandro Alvarado", Venezuela \\ Correo-e: zapager@yahoo.com
}

\begin{abstract}
Resumen-El presente trabajo de carácter cuantitativo, ofrece los resultados parciales de una investigación llevado a cabo en una población de medianas empresas del estado Lara, Venezuela, y que abarcó un grupo de variables de diseño organizativo ${ }^{1}$. En este caso, correspondió determinar los tipos básicos de trabajo que están presentes en las empresas objeto de estudio. Se parte de una revisión bibliográfica que permitió describir las características del trabajo y que luego se hicieron operativas mediante la aplicación de un cuestionario diseñado para tal fin. Los datos obtenidos en el trabajo de campo, fueron procesados a través de estadística multivariante. Entre los resultados se destaca la presencia de tres tipos básicos de trabajo: (a) complejo y sometido a cambios frecuentes, (b) complejo y no sometido a cambios frecuentes, $y$ (c) sencillo $y$ no sometido a cambios frecuentes.
\end{abstract}

Palabras clave - Entorno, medianas empresas, tipos básicos de trabajo, variables de diseño organizativo.

Abstract - This quantitative character paper, provides partial results of an investigation conducted in a medium-sized enterprises population Lara state, Venezuela, and that included a group of variables of organizational design. In this case, corresponds determine the basic types of work that are present in the medium-sized enterprises studied. Based on a literature review, was described the characteristics of work and it was operationalized by applying a questionnaire designed for that purpose. The data obtained in the field study, were processed through of multivariate statistical. Among the results emerged the presence of three basic types of work: (a) complex and subject to frequent changes, (b) complex and not subject to frequent changes, and (c) simple and not subject to frequent change.

Key Word - Environments, medium enterprises, basic types of work, organizational design variables.

\section{INTRODUCCIÓN}

\footnotetext{
${ }^{1}$ Centralización, formalización, especialización de tarea, sistema de incentivos y tipos básicos de trabajo.
}

La organización se considera un sistema complejo adaptativo, puesto que requiere adecuar de una forma no trivial su estructura interna con la complejidad de su entorno [1]. Es decir, tiene que encontrar los mecanismos y las vías necesarias para coordinar y ajustar sus componentes o variables internas con los componentes de su entorno o factores de contingencia. Esto con el propósito de tener mayores posibilidades de alcanzar los niveles de eficiencia y competitividad suficientes que le permitan mantenerse activa y así poder satisfacer tanto las demandas de su entorno, como lograr sus propias metas y objetivos. Ideas que provienen de los postulados de la teoría contingente [2], [3], [4], [5] y [6].

Dicha teoría, plantea que no existe una manera única de organización, toma la visión de que los factores contextuales, o de contingencia ${ }^{2}$, determinan el diseño y la naturaleza de los procesos y la estructura de la organización, con lo cual es necesario el ajuste de sus variables internas de diseño con esos condicionantes para que pueda alcanzar un desempeño superior [7], [2], [8], [9], [10] y [6]. Además, la organización también se encuentra influida por los factores del voluntarismo ${ }^{3}$, que se refiere a la acción y elección deliberada y discrecional de los directivos y gerentes que forma su visión frente a la concepción de cómo debe ser organizada y dirigida la empresa, y, por tanto, la concepción y elección de los bienes o servicios que debe producir [11], [12] y [10]. Constituyendo, pues, los factores del voluntarismo: la percepción del entorno, la discrecionalidad y grado de autonomía de los directivos, los estilos de dirección, los sesgos y mapas cognitivos, los valores y creencias, la experiencia y los conocimientos adquiridos. Configurando todo esto lo que se denomina la visión directiva [11], [13] y [10].

${ }^{2}$ Factores de contingencia tales como: el entorno, la tecnología, la estrategia, los conocimientos, los valores y el tamaño de la organización. ${ }^{3} \mathrm{El}$ énfasis sobre la capacidad del individuo y sus acciones en la determinación de lo que deberá ser y alcanzar la organización, se denomina en términos generales como el voluntarismo [10]. 
Esa situación conlleva a la organización a enfrentarse a múltiples problemas, algunos difíciles de entender y solucionar que responden a una elevada incertidumbre, mientras que otros pueden ser más sencillos de resolver y por tanto con respuestas organizativas de menor complejidad. Es por ello que requiere tanto de niveles adecuados de conocimientos y cualificación de sus miembros, como de recursos técnicos y operativos que le proporcionen las habilidades y capacidades necesarias para realizar el trabajo que le permita enfrentar y resolver las diferentes circunstancias a las que se enfrenta.

De ahí, surgen dos dimensiones del trabajo. Una primera, relacionada con el grado de dificultad o complejidad para comprender, analizar y ejecutar el trabajo, o lo que es lo mismo su grado de complejidad. Y una segunda dimensión, relacionada con las características cambiantes o no del trabajo, o grado de variedad, que puede implicar la ejecución de diferentes métodos, tareas o procedimientos que responden a la variedad o a los cambios en los bienes y servicios exigidos por el entorno, y que afectan el grado en el cual las tareas asociadas a esos trabajo pueden ser estructuradas, sistematizadas o programadas [14], [15], [13] y [16].

Esas dos dimensiones mencionadas son desarrolladas y combinadas en los trabajos de [14], [15], [13] y [16]. Surge de esa combinación cuatro tipos básicos de trabajo: (a) trabajo sencillo y no sometido a cambios frecuentes; (b) trabajo sencillo y sometido a cambios frecuentes; (c) trabajo complejo, cualificado y no sometido a cambios frecuentes, y (d) trabajo complejo, cualificado y sometido a cambios frecuentes.

De este modo, la presente investigación tiene como propósito obtener una tipología del trabajo a partir de la combinación de dos dimensiones: grado de complejidad del trabajo y grado de variedad del trabajo, en las medianas empresas del estado Lara, Venezuela.

Para lograr ese propósito, se desarrollan en el segundo apartado los fundamentos teóricos de los tipos básicos de trabajo tomado como referencia en esta investigación. En el tercer apartado, se aborda la metodología de investigación cuya naturaleza es de carácter cuantitativo. Seguidamente, en el cuarto apartado, se lleva a cabo el procesamiento y análisis de los datos mediante la aplicación de diferentes pruebas estadísticas multivariante. Por último, se presentan las principales conclusiones del estudio.

\section{MARCO TEÓRICO}

Como ha sido comentado, la tipología de tipos de trabajo utilizada aquí proviene de la propuesta desarrollada en [14], [15], [13] y [16], cuyo aporte inicial surge de los trabajos pioneros de [17] y [18]. La misma, está compuesta por cuatro tipos básicos de trabajo y cuyas características pueden tener una repercusión en el diseño e implementación de las variables estructurales de diseño organizativo tales como: centralización ${ }^{4}$ y la formalización ${ }^{5}$, y orienta sobre el tipo de entorno donde se desenvuelve la organización.

\section{Trabajo sencillo, rutinario y no sometido a cambios}

El trabajo toma estas características cuando la empresa se desenvuelve en un entorno que exige productos o servicios sencillos o fáciles de elaborar y que no están sometidos a cambios frecuentes; es decir, se corresponde con un escenario donde el entorno se caracteriza por ser estable y poco complejo y con bajos niveles de incertidumbre. En este caso, es posible poner en práctica la codificación y estandarización de los procesos de trabajo, lo que implica niveles elevados de formalización y especialización de tareas. Asimismo, debido a la naturaleza rutinaria y estable de las tareas, la dirección en los niveles superiores de la jerarquía puede retener la cantidad suficiente de conocimientos, información y experiencia para dirigir, controlar y supervisar directamente, y con cierta facilidad, las operaciones de la empresa. Estas circunstancias, dejan un marco claro para el establecimiento de altos niveles de centralización tanto estratégica como operática.

Asimismo, la ausencia en este modelo de tareas cualificadas y la falta de necesidad de gestión del conocimiento, hace que sean poco relevantes los valores compartidos y el compromiso como mecanismos de coordinación y control, el cual se lleva a cabo combinando, según los casos, diferentes niveles de formalización y centralización [10].

\section{Trabajo sencillo y sometido a cambios frecuentes}

Responde a un entorno que exige productos sencillos de elaborar pero sujetos a cambios frecuentes en sus características, lo que significa un entorno sencillo pero dinámico. En consecuencia, habrá un ligero aumento en la complejidad cognitiva ${ }^{6}$ en relación a la situación anterior, ya que a pesar de que las tareas son fáciles de entender se presenta una gran variedad en los productos elaborados por la empresa y por tanto en el trabajo a realizar. Situación que agrega un cierto grado de dificultad e incertidumbre y, en consecuencia, un nivel medio de complejidad cognitiva,

\footnotetext{
${ }^{4}$ Cuando la toma de decisiones es centralizada ésta recae en la alta dirección de la organización, cuando es descentralizada parte de la decisiones vinculada con el trabajo operativo y estratégico es delegada hacia los niveles medios e inferiores de la pirámide empresarial [7], [19], [20] y [21].

${ }^{5}$ Es el grado en el cual la organización depende de reglas, regulaciones, procedimientos escritos y estandarizados que predeterminan el comportamiento y las tareas de los empleados, y la toma de decisiones operativas [10], [22], [23] y [24].

${ }^{6}$ [25:119] señala que la complejidad cognitiva "surge de la necesidad de que los agentes -empleados y trabajadores- se enfrenten a determinados tipos de trabajo que requieren elevados niveles de cualificación y el reciclaje continuado de los conocimientos para desempeñar la tarea".
} 
puesto que lo que inicialmente es ausencia de complejidad cognitiva por el carácter analizable de los problemas y las tareas, se ve incrementada, en alguna medida, por la necesaria adaptación a los permanentes cambios. Esta situación, dificulta el uso intensivo de manuales, rutinas y procedimientos codificados o escritos, como mecanismos de coordinación y control del trabajo, habiendo, por tanto, una necesidad de mayor flexibilidad y de menos formalización.

De igual forma hay un incremento en los niveles de formación profesional, puesto que la presencia de una mayor variedad y cambios en los productos exige una mayor cualificación del personal que participa en las tareas o actividades de su diseño, dirección, supervisión y elaboración. En todo caso, en estas circunstancias "el producto o servicio concreto que debe elaborar la empresa modificará las proporciones entre formalización $(\mathrm{F})$ y centralización $(\mathrm{C})$, pero en general cabe suponer que para este tipo de trabajo tendremos $\mathrm{F}<\mathrm{C}$ ” [14:130].

Se observa que la gestión del trabajo y el estilo de dirección están orientados, en este caso, básicamente a una reducción del grado de formalización organizativa -control indirectoy a una mayor centralización en la toma de decisiones control directo- como mecanismo de control y coordinación. Sin embargo, la presencia de cambios y variedad en los productos exige del trabajador una mayor preparación y conocimientos que le provean de capacidad para enfrentarse a esa variedad.

\section{Trabajo complejo, cualificado y no sometido a cambios frecuentes}

La empresa se desenvuelve en un entorno complejo pero estable. Situación que exige poca variedad en los productos o servicios, pero con complejidad en sus características y por consiguiente complejidad para su elaboración. Aquí, se requiere de trabajos cualificados que tienen que ser llevados a cabo, principalmente, por individuos con un buen nivel de preparación profesional.

Debido a la estabilidad del entorno, se puede simplificar los requerimientos de gestión del conocimiento, puesto que estos se limitan, en parte, a los métodos y procedimientos establecidos por los administradores que integran el sistema de dirección, o a los métodos y procedimientos de aquellos profesionales de la base operativa de la empresa que participan directamente en el diseño, coordinación y producción de los bienes o servicios [15] y [14]. Esto significa que hay una mayor tendencia a la aplicación de una técnica conocida por una actividad profesional para resolver los problemas operativos, traducido, a su vez, en un mayor grado de formalización profesional ${ }^{7}$.

${ }^{7} \mathrm{La}$ formalización relacionada con los especialistas formados en instituciones de educación superior que se integran al sistema de dirección
De lo anterior se desprende entonces que los productos elaborados por la organización dependen de un alto nivel profesional. Deduciéndose que la presencia de formalización profesional, puede entrar en contradicción con los niveles de formalización organizativa. La empresa necesita establecer niveles mínimos de formalización organizativa en respuesta a la estabilidad del producto, pero con el cuidado de no entrar en conflicto con los intereses de la alta cualificación profesional que limitaría su acción y su capacidad para solucionar los problemas complejos generados de su trabajo. Por consiguiente, es necesario, y conveniente, aplicar un nivel medio de formalización organizativa y un nivel medio-alto formalización profesional. Resultando una formalización neta de un nivel medio-alto; aportada sobre todo por el alto grado de formalización profesional presente en esta situación de trabajo complejo y no sometido a cambios frecuentes.

Por otra parte, la formalización profesional y el grado medio-alto de complejidad cognitiva son contradictorios, también, con altos niveles de centralización puesto que los trabajos cualificados $-\mathrm{o}$ profesionales- requieren de suficientes niveles de autonomía e independencia para ejecutar su trabajo y de un poder adecuado para tomar decisiones. En otras palabras, es necesaria una elevada descentralización. De esta forma, entendemos que el control basado en la centralización es poco eficiente en la medida que existe un mayor nivel de profesionalización y complejidad cognitiva

\section{Trabajo complejo, cualificado y sometido a cambios frecuentes.}

Este tipo de trabajo se presenta en una organización que se desenvuelve en entorno complejo y dinámico lo cual genera una elevada incertidumbre en la realización del trabajo, siendo, por tanto, tareas de naturaleza poco programables y poco rutinarias. Esta parte del modelo implica un tipo de producto con un ritmo alto de cambio, variedad $\mathrm{y}$, por consiguiente, un alto requerimiento en la cantidad y calidad de conocimientos necesarios para materializarlos. Por lo tanto, ninguno de los dos mecanismos de coordinación y control basados en altos niveles de formalización organizativa o en la centralización puede aplicarse de manera satisfactoria para administrar el tipo de trabajo necesario aquí.

De este modo, la competitividad de la empresa depende fundamentalmente de su capacidad para enfrentar un entorno complejo y dinámico exigente de productos complejos y cambiantes en relación a sus diseños y

y operaciones de la empresa e incorporan estándares, métodos y procedimientos o formas de comportamiento, los cuales sirven de guía en la actuación del personal cualificado o que ejerce un trabajo profesional. 
procesos de elaboración, que requieren, a su vez, de modificación y desarrollo permanente del conocimiento ya existente [14], [15] y [13]. No obstante, la exigencia de personal cualificado para resolver problemas complejos puede introducir ciertos elementos de formalización en lo que se refiere a pautas profesionales que sirven de patrones de referencia para abordar la complejidad y la variedad en los productos, y por ende en el tipo de trabajo complejo. De esta manera, se estima la necesidad de una baja formalización organizativa y de un nivel medio-alto de formalización profesional.

En relación a la centralización, tampoco es efectiva y oportuna puesto que la alta complejidad cognitiva, producto del tipo de trabajo difícil de comprender y realizar exige mayor grado de cualificación, autonomía e iniciativa en los diferentes niveles de la jerarquía, de tal manera que se puedan aprovechar al máximo las capacidades y los conocimientos. Por tanto, los niveles de formalización organizativa y centralización serán reducidos; sin embargo, habrá un predominio de la formalización sobre la centralización debido a que las reglas y normas e incluso la estandarización de ciertas etapas del trabajo, son más compatibles con el trabajo cualificado y creativo [14] y [13].

Por otra parte, el carácter complejo y variado de los productos o servicios, y el tipo de trabajo complejo y dinámico requerido determinan la necesidad de formas de organización más orgánicas donde la mayoría de los mecanismos de control, coordinación y supervisión burocráticos -directos y algunos indirectos como la formalización- sean sustituidos por mecanismos más informales basados en la socialización, la adaptación mutua, el contacto directo y la participación.

\section{METODOLOGÍA DE LA INVESTIGACIÓN}

El presente trabajo con un carácter cuantitativo, ofrece los resultados parciales de un proyecto de investigación titulado: Las Variables de Diseño y su Relación con las Formas Organizativas que Adoptan las Medianas Empresas: Un Estudio Teórico y Empírico, subvencionado por el Consejo de Desarrollo Científico Humanístico, Tecnológico de la Universidad Centroccidental "Lisandro Alvarado", Venezuela.

El estudio de campo de esta investigación, se llevó a cabo sobre una población de 411 medianas empresas del estado Lara, Venezuela, cuya muestra final la constituyeron $61 \mathrm{de}$ esas empresas que contestaron válidamente los cuestionarios. Los mismos, fueron aplicados entre los meses de octubre de 2013 hasta junio de 2014, estando dirigidos a los directivos o a la alta gerencia de las empresas.

El cuestionario contiene, entre otras, las escalas de medición de los tipos de trabajo: complejo y variado, compuestas por múltiples ítems. Así, la escala de medición de la complejidad del trabajo inicialmente estaba conformada por cuatro ítems, pero del análisis de la fiabilidad resultó ser eliminado el ítems 2 , y la escala de medición de variedad del trabajo conformada por cinco ítems si continúo igual después de esa prueba. Se utilizó un escalamiento tipo Likert del 1 al 5 (muy desacuerdo=1, en desacuerdo $=2$, a veces $=3$, de acuerdo $=4$, muy de acuerdo $=5$ ).

La evaluación de la fiabilidad se llevó a cabo mediante el coeficiente o alpha de Cronbach. Este estadístico asume que la escala de medición está compuesta por elementos homogéneos que miden las mismas características, y su consistencia interna puede evaluarse mediante la correlación entre todos sus elementos o ítems [26]. Los resultados de esta prueba se presentan en la siguiente tabla 1

\begin{tabular}{lcc}
\hline $\begin{array}{c}\text { Variables } \\
\text { Tipos de Trabajos }\end{array}$ & $\begin{array}{c}\text { Cantidad de } \\
\text { Ítems }\end{array}$ & $\begin{array}{c}\text { Alpha de } \\
\text { Cronbach }\end{array}$ \\
\hline Trabajo Complejo & 3 & 0,679 \\
Trabajo Variado & 5 & 0,836 \\
\hline Tabla 1. Fiabilidad de las escalas de medición &
\end{tabular}

Se nota en la tabla 1 que el coeficiente alpha de Cronbach para la escala que mide el trabajo variado alcanza un valor de 0,836, que es mayor al valor mínimo de 0,700 recomendado por la literatura [27] y [28].

Con respecto a la escala que mide el grado de complejidad del trabajo, inicialmente se obtuvo una fiabilidad con un valor muy bajo, de 0,512 , procediéndose entonces a excluir el ítems 2 de acuerdo a la sugerencia de la prueba. Con esa decisión, se logró incrementar la fiabilidad a 0,679, todavía algo bajo pero más próximo al mínimo recomendado.

\section{ANALISIS DE LOS RESULTADOS}

Para dar respuesta al propósito de este trabajo, se aplicó en el tratamiento de los datos los métodos estadísticos multivariante de clasificación: análisis de conglomerados jerárquicos y de K-medias, acompañado con el ANOVA de un factor.

El análisis de conglomerados jerárquico proporcionó, a través del dendograma, el número de formas de organización. De este modo, se utilizó como variables de agrupación los ítems de las escalas utilizadas para medir los tipos de trabajo complejo y variado, aplicando el método de conglomeración de Ward y la medida de distancia de euclídea al cuadrado. Después de su análisis, se decidió que la solución natural para este caso era una clasificación de tres grupos de empresas (G1, G2 y G3).

Por su parte, el análisis de conglomerados de K-medias permitió obtener los valores de la media de cada variable evaluada en los grupos de tipos de trabajo. Esta última 
prueba, fue acompañada con un análisis de varianza para determinar si la media poblacional de las variables dependientes -trabajo complejo y trabajo variado- en cada nivel de la variable independiente -grupos de clasificación: G1, G2 y G3- difieren significativamente.

Así, los resultados obtenidos al aplicar e interpretar estas pruebas estadísticas se presenta en la tabla 2.

\begin{tabular}{|c|c|c|c|c|}
\hline $\begin{array}{l}\text { Tipos de } \\
\text { Trabajo }\end{array}$ & $\begin{array}{c}\text { Grupo 1 } \\
\text { (G1) } \\
\text { NE=23 } \\
(37,70 \%)\end{array}$ & $\begin{array}{c}\text { Grupo } 2 \\
\text { (G2) } \\
\text { NE=22 } \\
(36,07 \%)\end{array}$ & $\begin{array}{c}\text { Grupo } 3 \\
\text { (G3) } \\
\text { NE=16 } \\
(26,23 \%)\end{array}$ & $\begin{array}{c}\text { ANOVA } \\
\text { Sig. }\end{array}$ \\
\hline $\begin{array}{c}\text { Trabajo } \\
\text { Complejo }\end{array}$ & 3,93 & 4,00 & 2,90 & $\begin{array}{l}F=38,57 \\
\text { Sig }=0,00\end{array}$ \\
\hline $\begin{array}{l}\text { Trabajo } \\
\text { Variado }\end{array}$ & 3,91 & 2,68 & 2,73 & $\begin{array}{l}F=37,80 \\
\text { Sig }=0,00\end{array}$ \\
\hline
\end{tabular}

$\mathrm{NE}=$ representa el número de empresas por cada grupo formado

Tabla 2. Centro de los conglomerados finales

Ahora bien, el ANOVA de un Factor señala que los tipos de trabajo complejo y variado difieren significativamente en los grupos de empresas $(p=0,000)$, pero esto no quiere decir que todas sean distintas entre sí estadísticamente aunque tengan valores diferentes ${ }^{8}$. En ese sentido, se aplicó la prueba HSD de Tukey de comparación múltiple en el análisis de la varianza para determinar exactamente donde se ubicaban tales diferencias -ver tabla 3-. Cuestión que era necesaria realizar para poder caracterizar y clasificar a las empresas con la mayor precisión posible de acuerdo al tipo de trabajo que se realizan en ella.

\begin{tabular}{|c|c|c|c|c|}
\hline $\begin{array}{l}\text { Variable } \\
\text { dependiente }\end{array}$ & $\begin{array}{c}\text { Grupos } \\
\text { (I) }\end{array}$ & $\begin{array}{c}\text { Grupos } \\
(\mathbf{J})\end{array}$ & $\begin{array}{c}\text { Diferencia } \\
\text { de Medias } \\
\text { (I-J) }\end{array}$ & Sig. \\
\hline \multirow{6}{*}{ Trabajo Complejo } & 1 & 2 &,- 07246 & ,831 \\
\hline & & 3 & $1,03170(*)$ & 000 \\
\hline & 2 & 1 & ,07246 & ,831 \\
\hline & & 3 & $1,10417(*)$ & 000 \\
\hline & 3 & 1 & $-1,03170(*)$ & ,000 \\
\hline & & 2 & $-1,10417(*)$ & 000 \\
\hline \multirow{6}{*}{ Trabajo Variado } & 1 & 2 & $1,23123(*)$ & ,000 \\
\hline & & 3 & $1,18804(*)$ & 000 \\
\hline & 2 & 1 & $-1,23123(*)$ & 000 \\
\hline & & 3 &,- 04318 & ,966 \\
\hline & 3 & 1 & $-1,18804(*)$ &, 000 \\
\hline & & 2 & 04318 & 966 \\
\hline
\end{tabular}

Nota: * La diferencia de medias es significativa al nivel .05

Tabla 3. Prueba HSD de Tukey del ANOVA

De acuerdo a los resultados derivados del test de Tukey y presentados en la tabla 3, se desprende lo siguiente: (a) la media del tipo de trabajo complejo en el grupo tres (G3) cuyo valor es de 2,90, difiere significativamente de la media del grupo uno (G1) con 3,93 y del grupo dos con

\footnotetext{
${ }^{8}$ [29] nos recuerda que rechazar la hipótesis nula de igualdad de las medias entre los grupos no significa que todas las medias sean distintas entre sí, sino que al menos una es significativamente distinta de todas las demás.
}

4,00 (G2), pero estas no son estadística y significativamente diferentes entre sí, aunque sus valores absolutos son distintos. Por lo tanto, se considera a estos dos últimas grupos con media de valor alto y al grupo G3 con un valor bajo. (b) La media del tipo de trabajo variado en el grupo uno (G1) difiere estadística y significativamente de la media de los grupos dos y tres (G2 y G3), pero estos dos grupos no son estadística y significativamente diferentes entre sí, asignándoseles entonces a ambos un valor bajo y al grupo uno (G1) de alto.

En resumen, estos resultados permiten clasificar el tipo de trabajo para cada grupo de empresa formado. Cuestión que se presenta en la tabla 4, donde se ofrece la distribución de las empresas por grupo de tipos de trabajo formado.

\begin{tabular}{|c|c|c|c|}
\hline $\begin{array}{l}\text { Tipos de } \\
\text { Trabajo }\end{array}$ & $\begin{array}{c}\text { Grupo 1(G1) } \\
\text { NE=23 } \\
\text { Trabajo } \\
\text { Complejo } \\
\text { y Sometido } \\
\text { a Cambios } \\
\text { Frecuentes } \\
\end{array}$ & $\begin{array}{c}\text { Grupo 2(G2) } \\
\text { NE=22 } \\
\text { Trabajo } \\
\text { Complejo } \\
\text { y No Sometido } \\
\text { a Cambios } \\
\text { Frecuentes } \\
\end{array}$ & $\begin{array}{c}\text { Grupo3(G3) } \\
\text { NE=16 } \\
\text { Trabajo } \\
\text { Sencillo y No } \\
\text { Sometido } \\
\text { a Cambios } \\
\text { Frecuente } \\
\end{array}$ \\
\hline $\begin{array}{c}\text { Trabajo } \\
\text { Complejo }\end{array}$ & 3,93(alto) & 4,00 (alto) & 2,90 (bajo) \\
\hline $\begin{array}{l}\text { Trabajo } \\
\text { Variado }\end{array}$ & 3,91 (alto) & 2,68 (bajo) & 2,73 (bajo) \\
\hline
\end{tabular}

Nota: El sombreado indica que las variables en cada grupo no difieren significativamente

Tabla 4. Tipos de trabajo en las medianas empresas del estado Lara, Venezuela

La clasificación de tipos de trabajos presentada en la tabla 4 se obtiene de la siguiente manera: (a) Para las empresas del grupo uno (G1), su tipo de trabajo se caracteriza por ser complejo y a su vez sometido a cambios frecuentes puesto que la media de las variables trabajo complejo y trabajo variado tienen valores altos (3,93 y 3,91, respectivamente), dicho grupo lo conforman 23 empresas $(37,70 \%)$. (b) Las empresas del grupo dos (G2), que está constituido por 22 organizaciones $(36.07 \%)$, el tipo de trabajo se caracteriza por ser también complejo y no sometido a cambios frecuentes, puesto que la variable complejidad tiene una media de valor elevado $(4,00)$ y es baja para la variable trabajo variado $(2,68)$. Y (c) para el grupo tres, conformado por 16 empresas $(26,23 \%)$, el tipo de trabajo es sencillo y no sometido a cambios frecuentes puesto que tanto el valor de la media para el trabajo complejo y el trabajo variado es bajo (2,90 y 2,73 , respectivamente).

Se destaca el hecho de que el 73,77\% de las medianas empresas están ubicadas dentro de la dimensión de trabajos complejos, lo que refleja el entorno en el cual desarrollan sus actividades y las características de los productos que elaboran.

\section{CONCLUSIONES}


Como se indicó en la introducción, el objetivo del presente trabajo fue determinar y obtener una clasificación de los diferentes tipos de trabajo de acuerdo a los datos obtenidos en el estudio empírico, tomando como referencia dos dimensiones básicas del trabajo obtenidas a partir de la literatura consultada: la complejidad y la variedad del trabajo. Ambas dimensiones fueron evaluadas $\mathrm{y}$ operacionalizadas mediante los valores aportados por las medianas empresas encuestadas

De este modo, los resultados alcanzados permiten obtener evidencias empíricas de los distintos tipos de trabajo que se dan en las organizaciones, específicamente en las medianas empresas del estado Lara, Venezuela. Tipos de trabajos que pueden estar definidos por diversos factores tales como: (a) las naturaleza del entorno donde la organización desarrolla sus actividades y referido a su grado de complejidad, variedad e incertidumbre que emana de él; (b) las características técnicas y de calidad de los bienes o servicios que elabora la empresa o que exige su entorno; (c) los recursos y capacidades que posea la organización manifestado, por ejemplo, en: los recursos humanos, físicos, materiales, tecnológicos y financieros, y por el dominio y gestión adecuada del conocimiento; y (d) la visión directiva referida a la manera que concibe el propietario, el directivo o el gerente de cómo debe ser diseñada, organizada y dirigida la empresa.

Los resultados del estudio, señalan la presencia de tres tipos básicos de trabajo: (a) complejo y sometido a cambios frecuentes, (b) complejo y no sometido a cambios frecuentes, y (c) sencillo y no sometido a cambios frecuentes. Esto explica la variedad y los distintos grados de complejidad de los productos que elaboran las medianas empresas objeto de estudio de esta investigación y, en consecuencia, los diferentes entornos donde se desenvuelven.

Se destaca el hecho que un poco más del $70 \%$ de las medianas empresa, realizan trabajos complejos, independientemente de su variedad o dinamismo, lo que significa el grado de complejidad de las características de los productos que elaboran y por ende el nivel de dificultad en los procesos de producción. Esto, a su vez, implica trabajos cualificados que requieren de personal con un buen nivel de preparación profesional.

El tipo de trabajo sencillo y sometido a cambios frecuentes propuesto en el marco teórico, no aparece en la tipología obtenida del estudio de campo. Tal vez se encuentre incorporado entre los grupos 1 y 3 , cuya explicación, en parte, puede deberse a la posibilidad de que en las medianas empresas venezolanas hoy en día ya no compitan en entornos que, a la vez, tengan muy poca complejidad y mucha inestabilidad o variedad; es decir, aquella situación donde se requieren productos o servicios con cierta facilidad para comprender su proceso de elaboración pero sometidos a cambios frecuentes en sus características, o que la empresa tiene la necesidad con cierta periodicidad de realizar nuevas innovaciones en productos o servicios.

Se entiende entonces que los trabajos con bajos niveles de incertidumbre y en situaciones altamente predecibles llevan a establecer medidas relacionadas: (a) con procedimientos y reglas de trabajo bien claro y codificados, es decir, altos niveles de formalización; (b) con ámbitos de control generalmente amplios, lo que implica una alta centralización en la toma de decisiones principalmente a nivel estratégico y en muchos casos operativos; y (c) con áreas bien definidas de autoridad y responsabilidad. Por otra parte, otros trabajos no son rutinarios y no programados, por lo general con una amplia diversidad de tareas asociadas a procesos de conversión difícil de comprender y llevar a cabo, y que requerirán de bajos niveles de centralización y mayor participación -descentralización en la toma decisiones- y de una formalización organizativa más flexible y adecuada a las exigencias del personal cualificado que es necesario en este tipo de trabajo.

Finalmente, la presente investigación en lo particular ha tenido la intención de aportar evidencias empíricas que permitan seguir abriendo caminos para entender las características y las condiciones en las cuales se da el trabajo como un hecho fundamental para la vida económica y social de la organización. En lo general, se trata de seguir fortaleciendo los fundamentos teóricos que explican el diseño y el comportamiento de las organizaciones bajo un entorno cada vez más competitivo y exigente.

\section{REFERENCIAS}

[1]. M. Boisot and J. Child, "Organizations as adaptive system in complex environments: the case of china," Organization Science, vol. 10(3), pp. 237$232,1999$.

[2]. J. Child, Ch. Jeanne and D. Howard,"The performance of cross-border units in china: a test of natural selection, strategic choice and contingency theories,". Journal of International Business Studies, vol. 43(3), pp. 242-254, 2003.

[3] P. Garengo and U. Bititci, "Towards a contingency approach to performance measurement: an empirical study in scottish SMEs," International Journal of Operations \& Production Management, vol. 27(8), pp. 802-25, 2007.

[4]. H .Volberda, V. Niels, E. Verwaal, M.. Stienstra and A. Verdu, "Contingency fit, institutional fit, and firm performance: a Meta fit approach to organization-environment relationships," Organization Science, vol. 23(4), pp. 1040-1054, 2012 
[5]. K. Akingbola, "Contingency, fit and flexibility of hrm in nonprofit organizations," Employee Relations, 25(5), pp. 479-49, 2013.

[6]. G. J. Zapata Rotundo, "El determinismo en la teoría contingente: orígenes de sus fundamentos teóricos para su comprensión," Revista Ciencia y Sociedad, vol. 39(1), pp. 121-154, 2014.

[7]. L. Donaldson, The contingency theory of organizations, London: Sage Publications, 2001.

[8] C. Simon, "A configuration dorm of fit in management accounting contingency theory. an empirical investigation," The Business Review, Cambridge, Hollywood, vol. 7(2), pp. 220-227, 2007.

[9]. E. Pertusa-Ortega, J. Molina-Azorín y E. ClaverCortés, "Competitive strategy, structure and firm performance. A comparison of the resourcebased view and the contingency approach,". Management Decision, 48(8), pp. 1282-1303, 2010.

[10]. G. J. Zapata Rotundo y A. Mirabal, "Contingencia, voluntarismo y strategic Choice: un análisis teórico de sus enfoques sobre el entorno y el diseño de la organización," Revista Universidad y Empresa, vol. 15(34), pp. 119-139, 2013.

[11]. F. Peris, M. Peris-Ortiz y G.J. Zapata Rotundo, "Complementariedad de teorías en la administración eficiente del trabajo: un análisis microorganizativo," Compendium, vol. 9(17), pp. 75-94, 2006.

[12]. G. J. Zapata Rotundo y M. T. Canet Giner, "La Cognición del Individuo: Reflexiones sobre sus Procesos e influencia en la Organización," Revista Espacio Abierto: Cuaderno Venezolano de Sociología, vol. 18(2), pp. 46-62, 2009.

[13]. G. J. Zapata Rotundo, M. T. Canet Giner y F. Peris Bonet, "Visión directiva y elección estratégica: su influencia en los tipos básicos de trabajo," Cuadernos de Administración, vol. 21(37), pp.185-209, 2008.

[14]. F. Peris, T. González y M. Méndez, "Organización, diseño organizativo y comportamiento: modelo conceptual y modelo analítico," Quadern de Treball, 116 (nova época), 2001.

[15]. M. Moreno-Luzón, F. Peris, F. y T. González, La Gestión de la Calidad y Diseño de Organizaciones: Teoría y Estudio de Casos, España: Prentice Hall, 2001.

[16]. G. J. Zapata Rotundo, M. T. Canet Giner, "Tipos de trabajo y la formación de la especialización de tareas en la organización," Revista de Ciencias Sociales (RCS), vol. 18(1), pp. 58-73, 2012.

[17]. Ch. Perrow, "A framework form the Comparative Analysis of Organizations," American Sociological Review, vol. 32(2), pp. 194-208, 1967.

[18]. Ch. Perrow, Organizational Analysis: A Sociological View, Wadsworth Publishing, California, 1970.

[19]. A. Delić and E. Ahmetović, "Characteristics of organizational structure of bosnian and erzegovinian companies," Journal of Economics and Business, vol. XI(2), pp. 31-43, 2013.

[20]. J. Wynen, K. Verhoest and K. Rübecksen, "Decentralization in public sector organizations. do organizational autonomy and result control lead to decentralization toward lower hierarchical levels?," Public Performance \& Management Review, vol. 37(3), pp. 496-520, 2014.

[21]. S. Reichmann and S. Rohlfing-Bastian, "Decentralized task assignment and centralized contracting: on the optimal allocation of authority," Journal of Management Accounting Research, vol. 26(1), pp. 33-55, 2014.

[22]. J.Gibson, J. Ivancevich, J and J. Donnelly, Las organizaciones. comportamiento, estructura y procesos. McGraw-Hill, Décima Edición, 2001.

[23]. L. Pleshko, "Strategic orientation, organisational structure, and the associated effects on performance," Journal of Financial Services Marketing, vol. 12(1), pp. 53-64, 2007.

[24]. E. Hwang and M. Norton, "Environmental uncertainty, market-orientation strategy, and organizational structure in china's apparel retail stores," Advances in Business-Related Scientific Research Journal, vol. 5(1), pp. 1-12, 2014.

[25]. M. T. Canet Giner, El proceso de formación de la estrategia: su relación con la complejidad organizativa y los resultados. Análisis Aplicado al caso Español. Tesis Doctoral, 2001.

[26]. A. Pardo y M. Ruiz, SPSS 11. Guía para el Análisis de Datos, Madrid: McGraw-Hill Hispanoamericana, 2002.

[27]. J. Hair, R. Anderson, R. Tatham and W. Black, Análisis Multivariante, Madrid: Prentice Hall, 5ta edición, 1999.

[28]. N. Malhotra, N. (2004). Marketing Research: An Applied Orientation, Pearson Education, 4th ed.: Upper Saddle River (NJ), 2004. 
[29]. J. Aldás, Documentación del curso: metodología de investigación en dirección de empresas. programa de doctorado en dirección de empresas, Departamento de Dirección de Empresas "Juan José Renau Piqueras", Universitat de Valencia, 2001. 